\title{
Component Connectors with QoS Guarantees ${ }^{\star}$
}

\author{
Farhad Arbab, Tom Chothia, Sun Meng, Young-Joo Moon \\ CWI, Kruislaan 413, Amsterdam, The Netherlands \\ \{Farhad.Arbab, T.Chothia, Meng.Sun, Y.J.Moon\}@Cwi.nl
}

\begin{abstract}
Connectors have emerged as a powerful concept for composition and coordination of concurrent activities encapsulated as components and services. Compositional coordination models and languages serve as a means to formally specify and implement component and service connectors. They support largescale distributed applications by allowing construction of complex component connectors out of simpler ones. Modelling, analysis, and ensuring end-to-end Quality of Service (QoS) represent key concerns in such large-scale distributed applications. In this paper we introduce a compositional model of QoS, called Quantitative Constraint Automata, that reflects the underlying architecture of component/service composition represented by the Reo connector circuits. These can support compositional reasoning about component/service connectors, modelled as Reo circuits with QoS properties.

Keywords: Coordination, Composition, Reo, Quality of Service, Quantitative
\end{abstract} Constraint Automata

\section{Introduction}

Service-oriented computing (SOC) [14] is an emerging paradigm for the development of complex applications that may run on large-scale distributed systems. Such systems, which typically are heterogeneous and geographically distributed, usually exploit communication infrastructures whose topology frequently varies and components can, at any moment, connect to or detach from. Compositional coordination models and languages provide a formalisation of the "glue code" that interconnects the constituent components/services and organises the communication and cooperation among them in a distributed environment. They support large-scale distributed applications by allowing construction of complex component connectors out of simpler ones.

An example of such a model is Reo [2,6], which offers a powerful glue language for implementation of coordinating component connectors based on a calculus of channels. The work presented here adds Quality of Service to Reo, hence giving us a way of coordinating components that takes into account their costs and the costs of coordination.

Quality of Service (QoS) is a measure of the non-functional properties of services along multiple dimensions, such as reliability, security, scalability, response time, etc. Over the past few decades, several quantitative stochastic methods (e.g., stochastic Petri

\footnotetext{
* The work in this paper is supported by a grant from the GLANCE funding program of the Dutch National Organisation for Scientific Research (NWO), through project CooPer $(600.643 .000 .05 \mathrm{~N} 12)$.
} 
Nets [15], interactive Markov Chains [12] and different kinds of stochastic process algebras $[8,11,13])$ have been proposed and used in a variety of application areas to study different QoS metrics, mainly on performance evaluation. In recent years we have observed the phenomenon of unbundling of complex monolithic IT and communication services causing large-scale distributed applications to cross intra- and interorganisational borders. In such environments it becomes a great challenge to provide end-to-end QoS. A promising approach to meet this challenge advocates a compositional approach to provisioning QoS, which allows one to express connector QoS requirements as QoS requirements on the connector's constituent basic elements. However, extending connector specifications directly with information about non-functional concerns limits the reusability of the connector specification and hence any implementation of it. Thus there has been a great deal of interest recently in techniques to provide an effective separation of concerns for end-to-end non-functional requirements and the more stable functional requirements. Reo is a good candidate to serve as the base model for a calculus for compositional QoS, because it offers a fully compositional structure of architecturally meaningful user-defined primitives (i.e, channels) to construct systems with complex behavior, involving arbitrary combinations of synchronous and asynchronous protocols. Analytic or experimental QoS models for such structurally meaningful primitives are more likely to be available, robust, and application context independent (i.e., reusable).

QoS aspects have been handled by using constraint semirings (c-semirings for short) in $[9,20]$. Assuming a suitable level of abstraction for QoS constraints and a metric for the actual QoS values, c-semirings provide an algebraic structure with two operations, one to select among values and the other to combine values into a new $\mathrm{QoS}$ value. Thus compositionality of QoS values is guaranteed in this approach. Chothia and Kleijn extend c-semirings to an algebraic structure called Q-algebra [10], which have two operators for combining QoS values. In this way it becomes possible both to combine QoS values when they occur sequentially and also when they occur concurrently. Moreover, the QoS values can also be compared within the algebra. In general, QoS values are tuples where each component represents a particular aspect: the entries can be of different kinds (numerical to indicate latency, access rights of a service, memory usage, etc.) and a finite number of Q-algebras may be combined as a tuple with tuples as its values. The operators that calculate the combination of costs can be simple (e.g. addition or multiplication) giving an approximation of the true cost or much more complex, giving an accurate picture. The semiring framework, on which Q-algebras are based has been successfully used to model a range of real life situations [19]. Q-algebra is also used to build an automata model called Q-automata [10], which can be used to represent components or channels with costs.

The purpose of this paper is to introduce an operational model for reasoning about general QoS properties of the exogenous channel-based coordination language Reo [1, $2,4]$. An operational semantics of Reo connector circuits has been provided by constraint automata [6]. Extensions of constraint automata have been investigated for realtime constraints on behaviour of component connectors [3] and to study probabilistic and stochastic properties [5,7]. 
In this paper we consider the quality aspects of Reo circuits when the specification of channels and component interfaces can involve non-functional requirements. Because connectors, not components, are the primary concern in Reo, our primary interest here is with channels whose behaviour involves quality features and their composition. Remarkably, we do not refer to specific QoS aspects related to concrete services. On the contrary, we are concerned with a unifying theory of QoS model that might be used for different QoS measures. We introduce Quantitative Constraint Automata (QCA) as an extension to ordinary constraint automata with QoS values added as additional labels to the individual transitions indicating their use of resources, costs, reliabilities, etc. when executed. To support compositional reasoning, we provide semantic operators corresponding to Reo's main primitives (join and hiding) to model complex component connectors, and thus obtain a compositional framework to generate QCA from a given Reo circuit. Furthermore, we present several notions of simulation for QCA that serve to formalise the replacability of Reo circuits by means of refinement and cost reduction.

The rest of this paper is organised as follows: Section 2 contains a brief introduction to Reo and constraint automata. In Sections 3 and 4 we introduce QCA and show how Reo connectors are equipped with QoS characteristics. In Section 5 we present notions of simulation on QCA and their relationship. Section 6 concludes the paper.

\section{Reo and Constraint Automata}

In this section we provide a brief introduction to Reo [2], which is a channel-based exogenous coordination model wherein complex coordinators, called connectors, are compositionally built out of simpler ones. We summarise only the main concepts in Reo and its constraint automata semantics here. Further details about Reo and its semantics can be found in $[2,4,6]$.

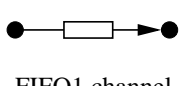

FIFO1 channel

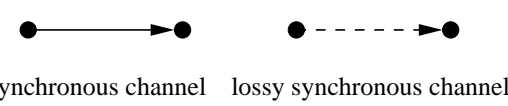

Fig. 1. Some Basic Channels in Reo

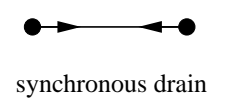

synchronous drain

Complex connectors in Reo are organised in a network of primitive connectors, called channels, that serve to provide the protocol that controls and organises the communication, synchronisation and cooperation among the components/services that they interconnect. Each channel has two channel ends. There are two types of channel ends: source and sink. A source channel end accepts data into its channel, and a sink channel end dispenses data out of its channel. It is possible for the ends of a channel to be both sinks or both sources. Reo places no restriction on the behaviour of a channel and thus allows an open-ended set of different channel types to be used simultaneously together. Each channel end can be connected to at most one component instance at any given time. Figure 1 shows the graphical representation of some simple channel types that will be used in this paper. A FIFO1 channel (FIFO1) represents an asynchronous channel with one buffer cell which is empty if no data item is shown in the box (as 
the one in Figure 1). If a data element $d$ is contained in the buffer of a FIFO1 channel then $d$ is shown inside the box in its graphical representation. A synchronous channel (Sync) has a source and a sink end and no buffer. It accepts a data item through its source end iff it can simultaneously dispense it through its sink. A lossy synchronous channel (LossySync) is similar to synchronous channel except that it always accepts all data items through its source end. The data item is transferred if it is possible for the data item to be dispensed through the sink end, otherwise the data item is lost. A synchronous drain (SyncDrain) has two source ends and no sink end. It can accept a data item through one of its ends iff a data item is also available for it to simultaneously accept through its other end as well.

Connectors are constructed by composing simpler ones via the join and hiding operations. Channels are joined together in a node which consists of a set of channel ends. Nodes are categorised into source, sink and mixed nodes, depending on whether all channel ends that coincide on a node are source ends, sink ends or a combination of the two. The hiding operation is used to hide the internal topology of a component connector. The hidden nodes can no longer be accessed or observed from outside.

A component can write data items to a source node that it is connected to. The write operation succeeds only if all (source) channel ends coincident on the node accept the data item, in which case the data item is transparently written to every source end coincident on the node. A source node, thus, acts as a replicator. A component can obtain data items, by an input operation, from a sink node that it is connected to. A take operation succeeds only if at least one of the (sink) channel ends coincident on the node offers a suitable data item; if more than one coincident channel end offers suitable data items, one is selected non-deterministically. A sink node, thus, acts as a non-deterministic merger. A mixed node non-deterministically selects and takes a suitable data item offered by one of its coincident sink channel ends and replicates it into all of its coincident source channel ends. Note that a component cannot connect to, take from, or write to mixed nodes. At most one component can be connected to a (source or sink) node at a time. The I/O operations are performed through interface nodes of components which are called ports.

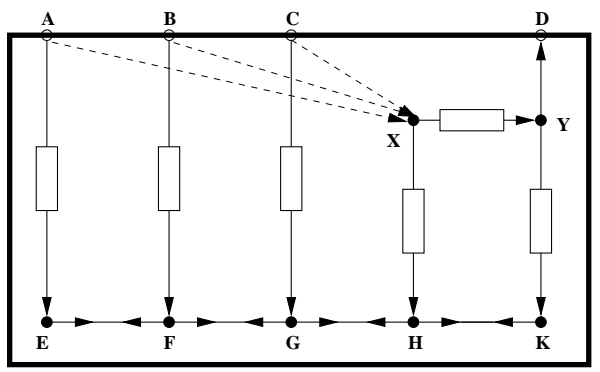

Fig. 2. Discriminator in Reo 
Example 1. Figure 2 shows a Reo connector that implements the behaviour of a blackbox that in the workflow literature is called a discriminator. The first item that arrives through one of the writers at nodes $\mathbf{A}, \mathbf{B}$ or $\mathbf{C}$ is selected for output through the taker at node $\mathbf{D}$. All three input values must arrive before the next cycle can start. Here, we use this figure to show the visual syntax for presenting Reo connector graphs. The enclosing thick box in this figure represents hiding: the topologies of the nodes (and their edges) inside the box are hidden and cannot be modified. Hiding yields a connector with a number of input/output ports, represented as nodes on the border of the bounding box, which can be used by other entities outside the box to interact with and through the connector. The basic channels used in the connector are synchronous channels (like the edge YD in Figure 2), lossy synchronous channels (e.g., AX, BX and $\mathbf{C X}$ in Figure 2), synchronous drain whose visual symbol appears as the edge EF, FG, GH or HK in Figure 2, and FIFO1 channel, like the edge AE in Figure 2.

Constraint automata (CA) [6] were introduced as a formalism to capture the operational semantics of Reo, based on timed data streams which also constitute the foundation of the coalgebraic semantics of Reo [4].

In the sequel, we assume a finite set $\mathscr{N}$ of nodes, and Data as a fixed, non-empty set of data that can be sent and received via channels. A data assignment denotes a function $\delta: N \rightarrow$ Data where $N \subseteq \mathscr{N}$. We use $D A(N)$ for the set of all data assignments for the node-set $N$. CA use a symbolic representation of data assignments by data constraints which are propositional formula built from the atoms " $d_{A} \in P$ ", " $d_{A}=d_{B}$ " or " $d_{A}=d$ " and standard Boolean connectors, where $A, B \in \mathscr{N}, d_{A}$ is a symbol for the observed data item at node $A$ and $d \in$ Data, $P \subseteq$ Data. We write $D C(N)$ to denote the set of data constraints that at most refer to the observed data items $d_{A}$ at node $A \in N$, and $D C$ for $D C(\mathscr{N})$. Logical implication induces a partial order $\leq$ on $D C: g \leq g^{\prime}$ iff $g \Rightarrow g^{\prime}$.

A constraint automaton over the data domain Data is a tuple $\mathscr{A}=\left(S, s_{0}, \mathscr{N}, \longrightarrow\right)$ where $S$ is a set of states, also called configurations, $s_{0} \in S$ is its initial state, $\mathscr{N}$ is a finite set of nodes, $\longrightarrow \subseteq \bigcup_{N \subseteq \mathscr{N}} S \times\{N\} \times D C(N) \times S$, called the transition relation. A transition fires if it observes data items in its respective ports/nodes of the component and according to the observed data, the automaton may change its state.

We write $s \stackrel{N, g}{\longrightarrow} s^{\prime}$ instead of $\left(s, N, g, s^{\prime}\right) \in \longrightarrow$ and refer to $N$ as the node-set and $g$ the guard for the transition. By an instance of $s \stackrel{N, g}{\longrightarrow} s^{\prime}$ we mean a transition of the form $s \stackrel{N, \delta}{\longrightarrow} s^{\prime}$ where $\delta$ is a data assignment for the nodes in $N$ with $\delta \models g$.

The intuitive operational behaviour of a constraint automaton can be specified by its runs. A run in a constraint automaton is defined as a (finite or infinite) sequence of consecutive transition instances

$$
r=s_{0} \stackrel{N_{0}, \delta_{0}}{\longrightarrow} s_{1} \stackrel{N_{1}, \delta_{1}}{\longrightarrow} s_{2} \stackrel{N_{2}, \delta_{2}}{\longrightarrow} \ldots
$$

We require that runs are either infinite or finite runs where the last state $s_{n}$ does not have any outgoing transition whose node set $N$ consists of mixed nodes only. This requirement can be understood as a maximal progress assumption for the mixed nodes.

Figure 3 shows the constraint automata for the basic channels given in Figure 1. Here and in the following, we skip the trivial guard true. 


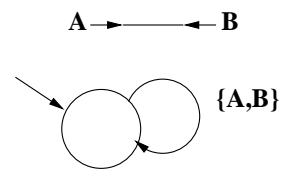

$$
\text { A }
$$

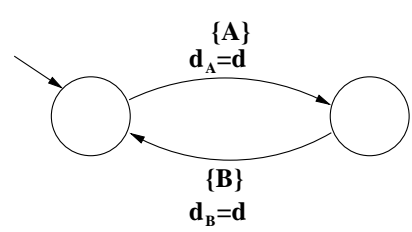

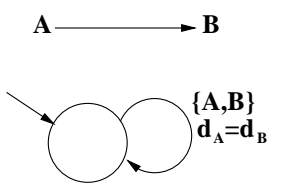

A $\ldots \ldots \ldots$

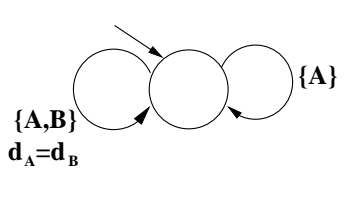

Fig. 3. Constraint Automata for Basic Reo Channels

Constructing complex connectors out of simpler ones is done by the join operation in Reo. Joining two nodes destroys both nodes and produces a new node on which all of their coincident channel ends coincide. Each channel in Reo, as well as the merger nodes are mapped to their own corresponding constraint automata. Reo's join operation can be realised by the product construction of constraint automata.

The product for two given constraint automata $\mathscr{A}_{1}=\left(S_{1}, s_{0,1}, \mathscr{N}_{1}, \longrightarrow_{1}\right)$ and $\mathscr{A}_{2}=\left(S_{2}, s_{0,2}, \mathscr{N}_{2}, \longrightarrow_{2}\right)$ is defined as a constraint automaton $\mathscr{A}_{1} \bowtie \mathscr{A}_{2}$ with the components

$$
\left(S_{1} \times S_{2},\left(s_{0,1}, s_{0,2}\right), \mathscr{N}_{1} \cup \mathscr{N}_{2}, \longrightarrow\right)
$$

where $\longrightarrow$ is given by the following rules:

$$
\begin{aligned}
& \text { - If } s_{1} \stackrel{N_{1}, g_{1}}{\longrightarrow} 1 s_{1}^{\prime}, s_{2} \stackrel{N_{2}, g_{2}}{\longrightarrow}{ }_{2} s_{2}^{\prime}, N_{1} \cap \mathscr{N}_{2}=N_{2} \cap \mathscr{N}_{1} \text { and } g_{1} \wedge g_{2} \text { is satisfiable, } \\
& \quad \text { then }\left\langle s_{1}, s_{2}\right\rangle \stackrel{N_{1} \cup N_{2}, g_{1} \wedge g_{2}}{\longrightarrow}\left\langle s_{1}^{\prime}, s_{2}^{\prime}\right\rangle . \\
& \text { - If } s_{1} \stackrel{N, g}{\longrightarrow} 1 s_{1}^{\prime} \text {, where } N \cap \mathscr{N}_{2}=\emptyset \text { then }\left\langle s_{1}, s_{2}\right\rangle \stackrel{N, g}{\longrightarrow}\left\langle s_{1}^{\prime}, s_{2}\right\rangle . \\
& \text { - If } s_{2} \stackrel{N, g}{\longrightarrow} 2 s_{2}^{\prime} \text {, where } N \cap \mathscr{N}_{1}=\emptyset \text { then }\left\langle s_{1}, s_{2}\right\rangle \stackrel{N, g}{\longrightarrow}\left\langle s_{1}, s_{2}^{\prime}\right\rangle .
\end{aligned}
$$

The first rule applies when there are two transitions in the automata that can fire together. This happens only if there is no shared name in the two automata that is present on one of the transitions but not present on the other one. In this case the transition in the resulting automaton has the union of the name sets on both transitions, and the data constraint is the conjunction of the data constraints of the two transitions. The second rule applies when a transition in one automaton can fire independently of the other automaton, which happens when the names on the transition are not included in the other automaton. The third rule is symmetric to the second one.

Another operator that is helpful for abstraction purposes and can be used in Reo to build connectors from networks is the hiding operator which declares the internal topology of the network as hidden. Hiding takes as input a constraint automaton $\mathscr{A}=\left(S, s_{0}, \mathscr{N}, \longrightarrow\right)$ and a non-empty node-set $M \subseteq \mathscr{N}$. The result is a constraint 
automaton hide $(\mathscr{A}, M)$ that behaves as $\mathscr{A}$ except that data flow at the nodes $A \in M$ is made invisible. Formally, hide $(\mathscr{A}, M)=\left(S, s_{0}, \mathscr{N} \backslash M, \longrightarrow_{M}\right)$ where $s \stackrel{\bar{N}, \bar{g}}{\longrightarrow} s^{\prime}$ iff there exists a transition $s \stackrel{N, g}{\longrightarrow} s^{\prime}$ such that $\bar{N}=N \backslash M$ and $\bar{g}=\exists M[g]$. Here $\exists M[g]$ stands short for $\bigvee_{\delta \in D A(M)} g\left[d_{A} / \delta . A \mid A \in M\right]$, where $g\left[d_{A} / \delta . A \mid A \in M\right]$ denotes the syntactic replacement of all occurrences of $d_{A}$ in $g$ for $A \in M$ with $\delta . A$. Therefore, $\exists M[g]$ formalises the set of data assignments for $\bar{N}$ that are obtained from a data assignment $\delta$ for $N$ where $g$ holds by dropping the assignments for the nodes in $N \cap M$.

\section{Quantitative Constraint Automata}

The basic channels presented in Section 2 assume a perfect behaviour of the channels. However, in real systems, certain channels might have different quality values, like the time of data transmission, reliability, throughput, etc. The behaviour of such channels cannot be captured by constraint automata. Several approaches have been developed to deal with different kinds of quality related properties in connectors $[3,5,7]$. In this section we introduce the notion of Quantitative Constraint Automata (QCA), which is an extension of constraint automata with Q-algebra and forms the basis for compositional specification and reasoning on Quality of Service (QoS) issues for connectors. A $Q$-algebra is an algebraic structure $R=(C, \oplus, \otimes, \oplus, \mathbf{0}, \mathbf{1})$ such that $R_{\otimes}=$ $(C, \oplus, \otimes, \mathbf{0}, \mathbf{1})$ and $R_{\oplus}=(C, \oplus, \Phi, \mathbf{0}, \mathbf{1})$ are both c-semirings. $C$ is a set of QoS values and is called the domain of $R$. The operation $\oplus$ induces a partial order $\leq$ on $C$, which is defined by $c \leq c^{\prime}$ iff $c \oplus c^{\prime}=c^{\prime}$. Much of the technical details of QCA is identical to or only slightly different than that of CA as presented in [6]. However, for completeness, we describe QCA in full detail here.

The states of a QCA stand for the network configurations, e.g., the contents of the buffers for FIFO channels. Each edge in a QCA is labelled with a tuple $(N, g, c)$ where $N$ is a set of ports (nodes) in a network where data-flow is observed simultaneously, $g$ is a Boolean condition on the observed data items, $c$ is a value in the domain $C$ of a Q-algebra that denotes the QoS or cost value, e.g., the (shortest) execution time, probability, bandwidth, etc.

We consider the quality of service for the basic channels in Reo by three values: $t$ (shortest time for data transmission), $c$ (allocated memory cost for the message transmission) and $p$ (reliability represented by the probability of successful transmission). The corresponding Q-algebras are given as:

- shortest time: $\left(\mathbb{R}_{+} \cup\{\infty\}, \max ,+, \max , \infty, 0\right)$

- memory cost: $\left(\mathbb{N}_{+} \cup\{\infty\}, \max ,+,+, \infty, 0\right)$

- reliability: $([0,1], \min , \times, \times, 0,1)$

Thus the corresponding QCA for the basic (quantitative) Reo channels SyncDrain, Sync, FIFO1 and LossySync used in this paper are given in Figure 4. For SyncDrain and Sync, the tuples labelled on the channels denote the corresponding quality of service for communication over these channels. For FIFO1, the tuple $\left(t_{3}, c_{3}, p_{3}\right)$ presents the QoS values for the operation to input a data to the buffer, and $\left(t_{4}, c_{4}, p_{4}\right)$ denotes the 

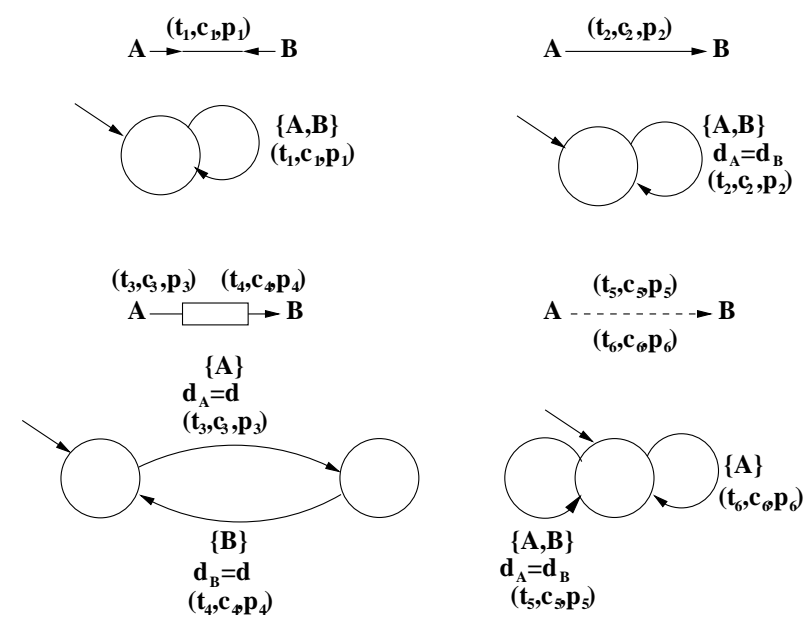

Fig. 4. QCAs for the basic Reo channels

QoS values for taking the data from the buffer. For LossySync, the tuple $\left(t_{5}, c_{5}, p_{5}\right)$ and $\left(t_{6}, c_{6}, p_{6}\right)$ present the QoS values for successful communication over the channel and information lost respectively.

Figure 5 shows on its left a quantitative Reo circuit consisting of three channels $\mathbf{A B}, \mathbf{A C}$ and $\mathbf{B C}$ which are of type SyncDrain, FIFO1 and Sync, respectively. The behaviour of this circuit can be seen as imposing an order on the flow of data items written to $\mathbf{A}$ and $\mathbf{B}$, through $\mathbf{C}$. The picture on the right shows a QCA corresponding to this quantitative Reo circuit. In the QCA on the right hand side in Figure 5, location $s_{0}$ stands for the initial configuration where the buffer is empty, while location $s(d)$ represents the configuration where the buffer contains a data element $d$. If node $\mathbf{C}$ is already for I/O operations in location $s(d)$ then we assume that $\mathbf{C}$ takes an element $d$ from the buffer and this corresponds to the transition labelled with the set $\{\mathbf{C}\}$, data constraint $d_{C}=d$ and the QoS values $t_{4}, c_{4}, p_{4}$ for the shortest time, memory cost and reliability values of the transition respectively. From the initial location $s_{0}$ we can input data from nodes $\mathbf{A}$ and $\mathbf{B}$ simultaneously, the data input at $\mathbf{A}$ will be stored in the buffer while the data input at $\mathbf{B}$ will be directly taken by $\mathbf{C}$ if the node $\mathbf{C}$ is ready to take it. The related QoS values are given as in the figure where $\left(t_{i}, c_{i}, p_{i}\right)$ represents the related QoS values for the basic channels, which are specified in Figure 4.

Similar to the work on constraint automata model, in QCA we use a finite set $\mathscr{N}$ of nodes and we do not distinguish between write and read operations at the nodes. We assume a fixed, non-empty and finite data domain Data consisting of the data items that can be transmitted through the channels. A data assignment denotes a function $\delta: N \rightarrow$ Data where $\emptyset \neq N \subseteq \mathscr{N}$. We use notations like $\delta=\left[A \mapsto \delta_{A}: A \in N\right]$ to describe the data-assignment that assigns the value $\delta_{A} \in$ Data to every node $A \in N$. Data constraints can be viewed as a symbolic representation of sets of data assignments. Formally data constraints are propositional formula built from the atoms " $d_{A}=d_{B}$ ", " $d_{A}=d$ " and " $d_{A} \in P$ " where $A, B$ are nodes, $d_{A}$ is a symbol for the observed data 

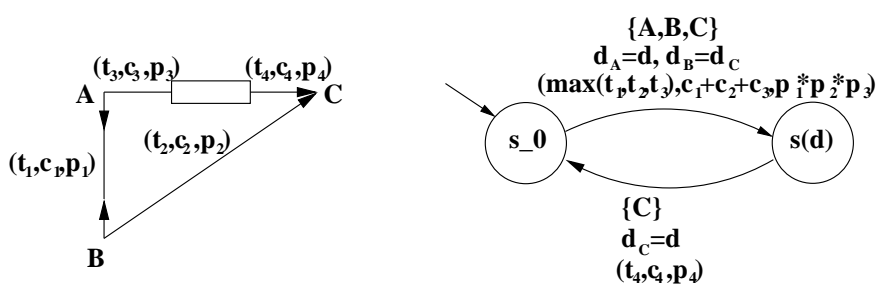

Fig. 5. A Reo Circuit and its Quantitative Constraint Automata

item at node $A$ and $d \in$ Data, $P \subseteq$ Data. For $N \subseteq \mathscr{N}, D A(N)$ denotes the set of all data assignments for the node-set $N$ and $D C(N)$ denotes the set of data constraints that at most refer to the terms $d_{A}$ for $A \in N$.

Definition 1. A Quantitative Constraint Automaton is a tuple $\mathscr{Q}=\left(S, s_{0}, \mathscr{N}, R, \longrightarrow\right)$ where

- $S$ is a set of states, also called configurations,

- $s_{0} \in S$ is its initial state,

- $\mathscr{N}$ is a finite set of nodes,

- $R=(C, \oplus, \otimes, \oplus, \mathbf{0}, \mathbf{1})$ is a labelled $Q$-algebra with domain $C$ of costs,

- $\subseteq \bigcup_{N \subseteq \mathscr{N}} S \times\{N\} \times D C(N) \times C \times S$, called the transition relation.

We write $q \stackrel{N, g, c}{\longrightarrow} p$ instead of $(q, N, g, c, p) \in \longrightarrow$ and refer to $N$ as the nodeset and $g$ the guard. Transitions where the node-set $N$ is non-empty are called visible, while transitions with the empty node-set are called hidden. In a hidden transition, none of the nodes is visible and the data constraints appear as unknown from outside, thus a hidden transition is witnessed only by its cost value. We denote hidden transitions by the label $\tau$ together with the cost. Moreover, a hidden transition cannot be carried out in cooperation with any other transitions. In effect, the ports of a hidden transition are no longer externally accessible to outside, but the costs of a hidden transition still affect the overall cost of a run. Each transition represents a set of possible interactions given by the transition instances that result by replacing the guard $g$ with a data assignment $\delta$ where $g$ holds. The QoS metric of a transition step is represented by the cost value $c$.

QCA composition is a binary function that maps two QCA with consistent Qalgebras into a new QCA. In the following definition we assume that the common nodes of the two QCAs are those where data flow must be synchronised.

Definition 2. For two $Q C A \mathscr{Q}_{1}=\left(S_{1}, s_{0,1}, \mathscr{N}_{1}, R, \longrightarrow_{1}\right)$ and $\mathscr{Q}_{2}=\left(S_{2}, s_{0,2}, \mathscr{N}_{2}, R\right.$, $\longrightarrow 2$ ) that have the same $Q$-algebra $R$, their product is defined as a $Q C A$

$$
\mathscr{Q}_{1} \bowtie \mathscr{Q}_{2}=\left(S_{1} \times S_{2},\left(s_{0,1}, s_{0,2}\right), \mathscr{N}_{1} \times \mathscr{N}_{2}, R, \longrightarrow\right)
$$

where $\longrightarrow$ is given by the following rules:

$$
\begin{aligned}
& \text { - If } s_{1} \stackrel{N_{1}, g_{1}, c_{1}}{\longrightarrow} 1 s_{1}^{\prime}, s_{2} \stackrel{N_{2}, g_{2}, c_{2}}{\longrightarrow} 2 s_{2}^{\prime}, N_{1} \cap \mathscr{N}_{2}=N_{2} \cap \mathscr{N}_{1} \neq \emptyset \text { and } g_{1} \wedge g_{2} \text { is } \\
& \text { satisfiable, then }\left\langle s_{1}, s_{2}\right\rangle \stackrel{N_{1} \cup N_{2}, g_{1} \wedge g_{2}, c_{1} \oplus c_{2}}{\longrightarrow}\left\langle s_{1}^{\prime}, s_{2}^{\prime}\right\rangle .
\end{aligned}
$$


- If $s_{1} \stackrel{N, g, c}{\longrightarrow} 1 s_{1}^{\prime}$, where $N \cap \mathscr{N}_{2}=\emptyset$ then $\left\langle s_{1}, s_{2}\right\rangle \stackrel{N, g, c}{\longrightarrow}\left\langle s_{1}^{\prime}, s_{2}\right\rangle$.

- If $s_{2} \stackrel{N, g, c}{\longrightarrow} 2 s_{2}^{\prime}$, where $N \cap \mathscr{N}_{1}=\emptyset$ then $\left\langle s_{1}, s_{2}\right\rangle \stackrel{N, g, c}{\longrightarrow}\left\langle s_{1}, s_{2}^{\prime}\right\rangle$.

A basic constraint automata can be "lifted" to a QCA by placing the null 1 value on each of its transitions. Therefore we can define the product of a quantitative and a non-quantitative constraint automata by applying this lifting and then the product, as defined above. We also note the product on QCA is associative.

The effect of hiding a node that is internal to some connector in a Reo circuit is that data flow at that node is no longer observable from outside. However, the quality/cost value should remain the same whether or not the node is hidden. Therefore, the hiding operator for QCA is defined as follows:

Definition 3. The hiding operator takes as input a $Q C A \mathscr{Q}=\left(S, s_{0}, \mathscr{N}, R, \longrightarrow\right)$ and a non-empty node-set $M \subseteq \mathscr{N}$. The result is a $Q C A$ hide $(\mathscr{Q}, M)$ that behaves as $\mathscr{Q}$ except that data flow at the nodes $A \in M$ is made invisible. Formally, hide $(\mathscr{Q}, M)=$ $\left(S, s_{0}, \mathscr{N} \backslash M, R, \longrightarrow M\right)$ where

- $q \stackrel{\bar{N}, \bar{g}, c}{\longrightarrow} M$ p iff there exists a transition $q \stackrel{N, g, c}{\longrightarrow} p$ such that $N \backslash M=\bar{N} \neq \emptyset$ and $\bar{g}=\exists M[g]$. Here $\exists M[g]$ stands short for $\bigvee_{\delta \in D A(M)} g\left[d_{A} / \delta . A \mid A \in M\right]$, where $g\left[d_{A} / \delta . A \mid A \in M\right]$ denotes the syntactic replacement of all occurrences of $d_{A}$ in $g$ for $A \in M$ with $\delta$. $A$.

- $q \stackrel{\tau, c}{\longrightarrow} M$ piff there exists a transition $q \stackrel{N, g, c}{\longrightarrow} p$ such that $N \backslash M=\emptyset$.

The automaton starts in an initial state. If the current state is $s$ then an instance $s \stackrel{N, \delta, c}{\longrightarrow} s^{\prime}$ of the outgoing transitions from $s$ is chosen, the corresponding I/O operations are performed and the next state is $s^{\prime}$. If there are several outgoing transitions from state $s$ the next transition is chosen non-deterministically. A formalisation of the possible (finite or infinite) observable data flow of a QCA is obtained by the notion of a run.

Definition 4. Let $s \in S$ be a state of a $Q C A \mathscr{Q}$. An s-run in $\mathscr{Q}$ denotes a (finite or infinite) sequence of consecutive transition instances

$$
r=s \stackrel{N_{0}, \delta_{0}, c_{0}}{\longrightarrow} s_{1} \stackrel{N_{1}, \delta_{1}, c_{1}}{\longrightarrow} s_{2} \stackrel{N_{2}, \delta_{2}, c_{2}}{\longrightarrow} \ldots
$$

An $s$-run is called initial if $s=s_{0}$. For finite runs we require that the last state $s^{\prime}$ does not have an outgoing hidden transition. This can be understood as a maximal progress assumption for hidden transitions, i.e., steps that do not require any interaction with the environment. The cost of an s-run $r$ is $\operatorname{cost}(r)=c_{0} \otimes c_{1} \otimes c_{2} \otimes \cdots$. A weak atomic run $r^{w a}=s \stackrel{N, \delta, c}{\rightarrow} s^{\prime}$ exists iff there is a finite run

$$
r=s_{0} \stackrel{\tau_{0}, c_{0}}{\longrightarrow} s_{1} \stackrel{\tau_{1}, c_{1}}{\longrightarrow} s_{2} \stackrel{\tau_{2}, c_{2}}{\longrightarrow} \ldots \stackrel{\tau_{n-2}, c_{n-2}}{\longrightarrow} s_{n-1} \stackrel{N, \delta, c_{n-1}}{\longrightarrow} s_{n}
$$

such that $s_{0}=s, s_{n}=s^{\prime}$ and $c_{0} \otimes c_{1} \otimes c_{2} \otimes \cdots \otimes c_{n-1}=c$. A weak run $r^{w}$ is defined analogously to a run as a sequence of weak atomic runs.

We use $R(\mathscr{Q})$ to denote the set of all runs of the QCA $\mathscr{Q}$, and $R_{0}(\mathscr{Q}) \subseteq R(\mathscr{Q})$ the set of all initial runs of $\mathscr{Q}$. 


\section{Quantitative Reo Circuits}

Reo is a channel-based exogenous coordination model wherein complex coordinators, called connectors, are built from instances of basic channel types using certain composition operators. In this paper, as in $[3,4,6]$, we concentrate on connectors that have graphical representations as Reo circuits which express the mechanisms that coordinate the data-flow through the channels connecting the input/output ports of some components, as well as the quality of service aspects of the behaviour.

Example 2. We now consider the example given in Figure 5 again. It depicts a connector which consists of three channels: $A B, A C$ and $B C$. These channels are of type SyncDrain, FIFO1 and Sync, respectively. According to the definition of the product operator, the composition of the corresponding QCA is given in Figure 5. We define the QoS values for the whole connector as: $t=\max \left(t_{1}, t_{2}, t_{3}\right)+t_{4}, c=\sum_{i=1}^{4} c_{i}$ and $p=\prod_{i=1}^{4} p_{i}$.

\begin{tabular}{|c|c|c|}
\hline & provider1 & provider2 \\
\hline SyncDrain & $\left(t_{1}=0, c_{1}=3, p_{1}=1\right)$ & $\left(t_{1}=0.1, c_{1}=2, p_{1}=1\right)$ \\
\hline Sync & $\left(t_{2}=1, c_{2}=2, p_{2}=0.95\right)$ & $\left(t_{2}=1, c_{2}=8, p_{2}=0.99\right)$ \\
\hline FIFO1 & $\left(t_{3}=1, c_{3}=2, p_{3}=0.9\right.$, & $\left(t_{3}=1, c_{3}=5, p_{3}=1\right.$, \\
& $\left.t_{4}=0.5, c_{4}=2, p_{4}=0.9\right)$ & $\left.t_{4}=1, c_{4}=5, p_{4}=0.99\right)$ \\
\hline LossySync & $\left(t_{5}=1, c_{5}=2, p_{5}=0.95\right.$, & $\left(t_{5}=1, c_{5}=3, p_{5}=0.99\right.$, \\
& $\left.t_{6}=0.1, c_{6}=1, p_{6}=0.95\right)$ & $\left.t_{6}=0.2, c_{6}=0.5, p_{6}=0.99\right)$ \\
\hline
\end{tabular}

Fig. 6. The QoS values for the basic channels

We may have different implementations for the Reo basic channels (and connectors). These implementations may have different QoS properties. For instance, the table in Figure 6 presents the QoS values for every basic channel in the Reo circuit of Figure 5 offered by two different service providers. Using these offerings, the QoS values for the Reo circuit are computed as follows:

- provider1: $t=1.5, c=9, p=0.7695$;

- provider2: $t=2, c=20, p=0.9801$.

Suppose now that a client's QoS requirement of this connector states that the cost of the whole connector (as defined above) should be no more than 15 units and the probability of successful transmission should be greater than 90 percent. Neither of the above two alternatives meet these requirements. However, we can choose the Sync offered by provider1, and the SyncDrain and the FIFO1 offered by provider2, and compose them together. Now the QoS value of the whole connector are: $t=2, c=14, p=0.9405$, which satisfies the requirement.

Example 3. We now show a more interesting example: the discriminator connector in Reo as given in Figure 2. The basic channel types SyncDrain, Sync, FIFO1 and 


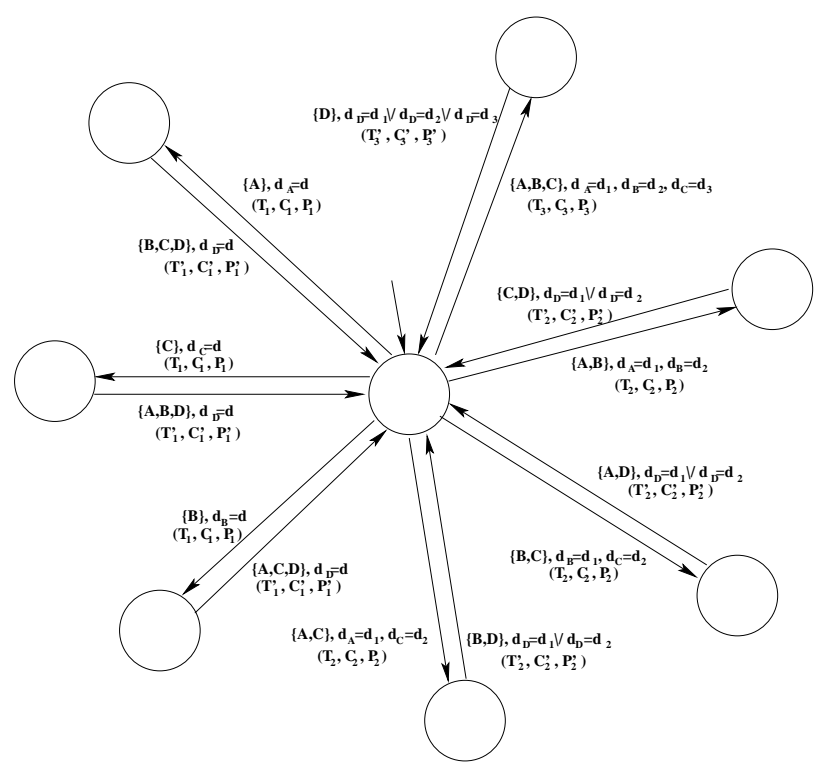

Fig. 7. The QCA for the discriminator connector in Reo

LossySync used in this example are all equipped with QoS labels. The corresponding QCAs and the QoS values for basic channels offered by different providers are given in Figures 4 and 6, respectively. The resulting QCA after composition and hiding all of its internal nodes appears in Figure 7, where its QoS properties are given in Figure $8{ }^{1}$.

The QoS value for the QCA in Figure 7 using the offerings of our two providers in Figure 6 are, for provider 1 :

$$
\begin{aligned}
& T_{1}=T_{2}=T_{3}=1, T_{1}^{\prime}=T_{2}^{\prime}=T_{3}^{\prime}=2, \\
& C_{1}=8, C_{2}=12, C_{3}=16, C_{1}^{\prime}=34, C_{2}^{\prime}=33, C_{3}^{\prime}=30, \\
& P_{1} \approx 0.69, P_{2} \approx 0.48, P_{3} \approx 0.5, P_{1}^{\prime} \approx 0.33, P_{2}^{\prime} \approx 0.39, P_{3}^{\prime} \approx 0.41
\end{aligned}
$$

and for provider2:

$$
\begin{aligned}
& T_{1}=T_{2}=T_{3}=1, T_{1}^{\prime}=T_{2}^{\prime}=T_{3}^{\prime}=3.1, \\
& C_{1}=18, C_{2}=26, C_{3}=34, C_{1}^{\prime}=62, C_{2}^{\prime}=61.5, C_{3}^{\prime}=56, \\
& P_{1}=0.99, P_{2} \approx 0.98, P_{3} \approx 0.97, P_{1}^{\prime} \approx 0.89, P_{2}^{\prime} \approx 0.89, P_{3}^{\prime} \approx 0.92
\end{aligned}
$$

As in the previous example, we may have some QoS requirements for the connector that may not be satisfied by either of the above alternatives, but it may be possible to select appropriate basic channels from different providers separately and compose them together to satisfy them, we skip the details here.

\footnotetext{
${ }^{1}$ In fact the QCA given in Figure 7 is not exactly the QCA resulting from composition and hiding. There are many more states and transitions in the resulting QCA, but most of the extra transitions are $\tau$-transitions. Therefore, we merge them with the visible transitions together as weak atomic runs of Definition 4 and represent them simply as single transitions in Figure 7.
} 


\begin{tabular}{|c|c|}
\hline$T_{1}=\max \left\{t_{3}, t_{5}\right\}$ & $T_{1}^{\prime}=\max \left\{t_{2}, t_{3}, t_{3}+t_{4}, t_{6}\right\}+\max \left\{t_{4}, t_{1}+t_{4}\right\}$ \\
\hline$C_{1}=3 \times c_{3}+c_{5}$ & $C_{1}^{\prime}=4 \times c_{1}+c_{2}+3 \times c_{3}+6 \times c_{4}+2 \times c_{6}$ \\
\hline$P_{1}=p_{3}^{3} \times p_{5}$ & $P_{1}^{\prime}=p_{1}^{4} \times p_{2} \times p_{3}^{3} \times p_{4}^{6} \times p_{6}^{2}$ \\
\hline$T_{2}=\max \left\{t_{3}, t_{5}\right\}$ & $T_{2}^{\prime}=\max \left\{t_{2}, t_{3}, t_{3}+t_{4}, t_{6}\right\}+\max \left\{t_{4}, t_{1}+t_{4}\right\}$ \\
\hline$C_{2}=4 \times c_{3}+2 \times c_{5}$ & $C_{2}^{\prime}=4 \times c_{1}+c_{2}+2 \times c_{3}+7 \times c_{4}+c_{6}$ \\
\hline$P_{2}=p_{3}^{4} \times p_{5}^{2}$ & $P_{2}^{\prime}=p_{1}^{4} \times p_{2} \times p_{3}^{2} \times p_{4}^{7} \times p_{6}$ \\
\hline$T_{3}=\max \left\{t_{3}, t_{5}\right\}$ & $T_{3}^{\prime}=\max \left\{t_{2}, t_{3}+t_{4}\right\}+\max \left\{t_{4}, t_{1}+t_{4}\right\}$ \\
\hline$C_{3}=5 \times c_{3}+3 \times c_{5}$ & $C_{3}^{\prime}=4 \times c_{1}+c_{2}+c_{3}+7 \times c_{4}$ \\
\hline$P_{3}=p_{3}^{5} \times p_{5}^{3}$ & $P_{3}^{\prime}=p_{1}^{4} \times p_{2} \times p_{3} \times p_{4}^{7}$ \\
\hline
\end{tabular}

Fig. 8. The QoS values for the discriminator

\section{Simulation on QCA}

Simulation relations were first introduced by Milner [18] for the purpose of comparing programs, and widely used later to show abstraction and refinement between models and specifications. They provide a sufficient condition for language inclusion that can be established with low complexity, and their precongruence properties are suited for compositional reasoning. In [6] simulation relations for ordinary constraint automata were defined to verify if two automata are language equivalent or the language of one is contained in the language of the other. In this section we propose to use quality improving simulation as a way to guarantee not only the inclusion of languages induced by Reo circuits, but also a higher (or at least equal) quality. For example, we may ask a connector implementation to be always more reliable, or faster than what is required by the specification, where both the specification and the implementation are given as QCA.

Before the definition of simulation, we first define some notation. For a QCA $\mathscr{Q}=$ $\left(S, s_{0}, \mathscr{N}, R, \longrightarrow\right)$, given a relation $\preceq \subseteq S \times S$ and a set of states $P \subseteq S, P$ is called $\preceq$-upward closed iff for all states $p \in P$ and $p \preceq p^{\prime}, p^{\prime} \in P$. For a relation $\preceq$ and two $\preceq$-upward closed sets $P_{1}$ and $P_{2}$, if there exist $s_{1} \in P_{1}$ and $s_{2} \in P_{2}$, such that $s_{1} \preceq s_{2}$, then $P_{1} \cup P_{2}$ is also $\preceq$-upward closed. Furthermore, let $s \in S$ be a state in $\mathscr{Q}, N \subseteq \mathscr{N}$ and $P \subseteq S$, then

$$
\begin{aligned}
d c(s, N, P) & =\bigvee\left\{g: s \stackrel{N, g, c}{\longrightarrow} s^{\prime} \text { for some } s^{\prime} \in P\right\} \\
d c_{w}(s, N, P) & =\bigvee\left\{g: s \stackrel{N, g, c}{\longrightarrow} s^{\prime} \text { for some } s^{\prime} \in P\right\}
\end{aligned}
$$

and

$$
\begin{aligned}
\operatorname{cost}\left(s, N, g, s^{\prime}\right) & =\left\{c: s \stackrel{N, g, c}{\longrightarrow} s^{\prime} \text { for some } s^{\prime} \in P\right\} \\
\operatorname{cost}_{w}\left(s, N, g, s^{\prime}\right) & =\left\{c: s \stackrel{N, g, c}{\longrightarrow} s^{\prime} \text { for some } s^{\prime} \in P\right\}
\end{aligned}
$$

Note that by introducing upward closed set, we can make an abstraction on QCA and lead to a smaller state space. Here $d c(s, N, P)\left(d c_{w}(s, N, P)\right.$ respectively) denotes 
the weakest data constraint that ensures the existence of an $N$-transition (a weak atomic run with node set $N$ respectively) from $s$ to $P$. We now recall the definition of simulation for ordinary constraint automata [6] and give the following definition for QCA. Simulation of QCA considers only the functional aspects of connectors and ignores their quality aspects.

Definition 5. Let $\mathscr{Q}=\left(S, s_{0}, \mathscr{N}, R, \longrightarrow\right)$ be a $Q C A$ and $\preceq$ a reflexive binary relation on $S . \preceq$ is called a simulation for $\mathscr{Q}$ if for all pairs $\left(s_{1}, s_{2}\right) \in \preceq$, all $\preceq$-upward closed sets $P \subseteq S$ and every $N \subseteq \mathscr{N}$ :

$$
d c\left(s_{1}, N, P\right) \leq d c\left(s_{2}, N, P\right)
$$

A state $s_{1}$ is simulated by another state $s_{2}$ (or $s_{2}$ simulates $s_{1}$ ), denoted as $s_{1} \preceq s_{2}$, iff there exists a simulation relation $\preceq$ with $\left(s_{1}, s_{2}\right) \in \preceq$. A QCA $\mathscr{Q}_{2}$ simulates another QCA $\mathscr{Q}_{1}$ (denoted as $\mathscr{Q}_{1} \preceq \mathscr{Q}_{2}$ ) iff the initial state of $\mathscr{Q}_{1}$ is simulated by the initial state of $\mathscr{Q}_{2}{ }^{2}$.

The above notion of simulation is some kind of "strong relation" because it refers to the stepwise behaviour, but does not accumulate the effect of non-observable behaviour alternatives. Thus, we define a notion of weak simulation for QCA analogously to simulation over weak atomic runs as follows:

Definition 6. For a $Q C A \mathscr{Q}=\left(S, s_{0}, \mathscr{N}, R, \longrightarrow\right)$, and a reflexive binary relation $\preceq_{w}$ on $S$, $\preceq_{w}$ is called a weak simulation if for all pairs $\left(s_{1}, s_{2}\right) \in \preceq_{w}$, all $\preceq_{w}$-upward closed sets $P$ and every $N \subseteq \mathscr{N}$,

$$
d c_{w}\left(s_{1}, N, P\right) \leq d c_{w}\left(s_{2}, N, P\right)
$$

Next, we introduce our notion of quality improving simulation (QIS). We distinguish two classes of QISes: QIS and Weak QIS. Quality improving simulations impose conditions over the cost values corresponding to the quality of each transition separately. In contrast, weak quality improving simulations constrain the cost values corresponding to the weak atomic runs. The definitions are as follows:

Definition 7. Let $\mathscr{Q}=\left(S, s_{0}, \mathscr{N}, R, \longrightarrow\right)$ be a $Q C A$ and $\lesssim \subseteq S \times S$ a reflexive binary relation on the state space of $\mathscr{Q}$. $\lesssim$ is called a quality improving simulation relation iff $\lesssim$ is a simulation and for all pairs $\left(s_{1}, s_{2}\right) \in \lesssim$,

$$
\forall c \in \operatorname{cost}\left(s_{1}, N, g, s_{1}^{\prime}\right) . \exists c^{\prime} \in \operatorname{cost}\left(s_{2}, N, g, s_{2}^{\prime}\right) . s . t . s_{1}^{\prime} \lesssim s_{2}^{\prime} \wedge c \leq c^{\prime}
$$

A state $s_{1}$ is quality improving simulated by another state $s_{2}$, denoted as $s_{1} \lesssim s_{2}$, iff there exists a quality improving simulation relation $\lesssim$ with $\left(s_{1}, s_{2}\right) \in \lesssim$. A QCA $\mathscr{Q}_{2}$ quality improving simulates another $Q C A \mathscr{Q}_{1}$ (denoted as $\mathscr{Q}_{1} \lesssim \mathscr{Q}_{2}$ ) iff the initial state of $\mathscr{Q}_{1}$ is quality improving simulated by the initial state of $\mathscr{Q}_{2}$.

\footnotetext{
${ }^{2}$ Here and in Definition $7 \mathscr{Q}_{1}$ and $\mathscr{Q}_{2}$ should rely on the same set of names, and be combined into a "large" QCA obtained through the disjoint union of the state spaces of $\mathscr{Q}_{1}$ and $\mathscr{Q}_{2}$.
} 
Example 4. Let's go back to Example 2. For the two providers, we have two concrete QCAs $\mathscr{Q}_{1}$ and $\mathscr{Q}_{2}$ respectively for the QCA given in Figure 5. In other words, the two QCAs have same behavior but different QoS values. Suppose we only care about the timing performance on the communication with this connector, then we can get the following table focusing on the time value costs. In this table each state is renamed like $s x_{i}$ where $i$ represents the corresponding QCA and $s x$ represents the derived state name in Figure 5.

\begin{tabular}{|l|l|}
\hline $\mathscr{Q}_{1}$ & $\mathscr{Q}_{2}$ \\
\hline$d c\left(s 0_{1},\{A, B, C\},\left\{s(d)_{1}, s(d)_{2}\right\}\right)=$ & $d c\left(s 0_{2},\{A, B, C\},\left\{s(d)_{1}, s(d)_{2}\right\}\right)=$ \\
$\left\{d_{A}=d \wedge d_{B}=d\right\}$ & $\left\{d_{A}=d \wedge d_{B}=d\right\}$ \\
\hline $\operatorname{cost}\left(s 0_{1},\{A, B, C\}\right.$, & $\operatorname{cost}\left(s 0_{2},\{A, B, C\}\right.$, \\
$\left.d_{A}=d \wedge d_{B}=d, s(d)_{1}\right)=\{1\}$ & $\left., d_{A}=d \wedge d_{B}=d, s(d)_{2}\right)=\{1\}$ \\
\hline$d c\left(s(d)_{1},\{C\},\left\{s 0_{1}, s 0_{2}\right\}\right)=\left\{d_{C}=d\right\}$ & $d c\left(s(d)_{2},\{C\},\left\{s 0_{1}, s 0_{2}\right\}\right)=\left\{d_{C}=d\right\}$ \\
\hline $\operatorname{cost}\left(s(d)_{1},\{C\}, d_{C}=d, s 0_{1}\right)=\{0.5\}$ & $\operatorname{cost}\left(s(d)_{2},\{C\}, d_{C}=d, s 0_{2}\right)=\{1\}$ \\
\hline
\end{tabular}

By defining relation $\lesssim=\left\{\left(s 0_{1}, s 0_{2}\right),\left(s(d)_{1}, s(d)_{2}\right)\right\}$, we can find that it is a quality improving simulation, since we have the condition

$$
d c\left(s 0_{1},\{A, B, C\},\left\{s(d)_{1}, s(d)_{2}\right\}\right) \leq d c\left(s 0_{2},\{A, B, C\},\left\{s(d)_{1}, s(d)_{2}\right\}\right)
$$

and

$$
d c\left(s(d)_{1},\{C\},\left\{s 0_{1}, s 0_{2}\right\}\right) \leq d c\left(s(d)_{2},\{C\},\left\{s 0_{1}, s 0_{2}\right\}\right)
$$

Furthermore, the condition in Definition 7 is also satisfied. Therefore, we can conclude that $\mathscr{Q}_{1} \lesssim \mathscr{Q}_{2}$.

Definition 8. Weak quality improving simulation is defined analogously to quality improving simulation over weak atomic runs. For a $Q C A \mathscr{Q}=\left(S, s_{0}, \mathscr{N}, R, \longrightarrow\right)$, and a reflexive binary relation $\lesssim_{w q}$ on $S, \lesssim_{w q}$ is called a weak quality improving simulation iff $\lesssim w q$ is a weak simulation and for all pairs $\left(s_{1}, s_{2}\right) \in \lesssim_{w q}$,

$$
\forall c \in \operatorname{cost}_{w}\left(s_{1}, N, g, s_{1}^{\prime}\right) . \exists c^{\prime} \in \operatorname{cost}_{w}\left(s_{2}, N, g, s_{2}^{\prime}\right) . \text { s.t. } s_{1}^{\prime} \lesssim w q s_{2}^{\prime} \wedge c \leq c^{\prime}
$$

The different types of simulation introduced in this section are ordered with respect to how closely they distinguish behaviour alternatives of QCA.

Proposition 1. Simulation relations satisfy the following partial order:

$$
\begin{aligned}
\mathscr{Q}_{1} & \lesssim \mathscr{Q}_{2} \Rightarrow \mathscr{Q}_{1} \preceq \mathscr{Q}_{2} \\
\mathscr{Q}_{1} \lesssim \mathscr{Q}_{2} & \Rightarrow \mathscr{Q}_{1} \lesssim w q \mathscr{Q}_{2} \\
\mathscr{Q}_{1} \lesssim w q \mathscr{Q}_{2} & \Rightarrow \mathscr{Q}_{1} \preceq_{w} \mathscr{Q}_{2} \\
\mathscr{Q}_{1} \preceq \mathscr{Q}_{2} & \Rightarrow \mathscr{Q}_{1} \preceq_{w} \mathscr{Q}_{2}
\end{aligned}
$$

In the context of Reo circuits, the connectors of practical interest are mostly composed from a family of simpler connectors. A compositional approach to modelling and analysis of such a connector is based on the description of the sub-connectors, without further information about the composed connector. As usual, compositionality is captured by the following definition: 
Definition 9. A relation $\lesssim$ between automata is compositional iff

$$
\mathscr{P}_{1} \lesssim \mathscr{Q}_{1} \wedge \mathscr{P}_{2} \lesssim \mathscr{Q}_{2} \Rightarrow \mathscr{P}_{1} \bowtie \mathscr{P}_{2} \lesssim \mathscr{Q}_{1} \bowtie \mathscr{Q}_{2}
$$

The following theorem provides a congruence result for the first three simulation preorders with respect to the product operator. This result allows us to refine a QCA by another one that may improve its quality without affecting its behaviour. However, the compositionality does not hold for the weak quality improving simulation.

Theorem 1. Simulation, quality improving simulation and weak simulation are compositional with respect to the product operation.

Proof (sketched): We consider the relation $\mathscr{R}=\left\{\left(\left\langle s_{1}, s_{2}\right\rangle,\left\langle s_{1}^{\prime}, s_{2}^{\prime}\right\rangle\right): s_{1} \preceq s_{1}^{\prime}, s_{2} \preceq\right.$ $\left.s_{2}^{\prime}\right\}$ and show that it is a simulation (quality improving simulation and weak simulation respectively). For simulation, we have

$$
d c\left(\left\langle s_{1}, s_{2}\right\rangle, N, P\right)=\bigvee\left(d c_{\mathscr{P}_{1}}\left(s_{1}, N_{1}, P\right) \wedge d c_{\mathscr{P}_{2}}\left(s_{2}, N_{2}, P\right)\right)
$$

and

$$
d c\left(\left\langle s_{1}^{\prime}, s_{2}^{\prime}\right\rangle, N, P\right)=\bigvee\left(d c_{\mathscr{Q}_{1}}\left(s_{1}^{\prime}, N_{1}, P\right) \wedge d c_{\mathscr{Q}_{2}}\left(s_{2}^{\prime}, N_{2}, P\right)\right)
$$

where $N_{1} \cup N_{2}=N$. Since $s_{1} \preceq s_{1}^{\prime}, s_{2} \preceq s_{2}^{\prime}, d c \mathscr{P}_{i}\left(s_{i}, N_{i}, P\right) \leq d c_{\mathscr{Q}_{i}}\left(s_{i}^{\prime}, N_{i}, P\right)$, thus $d c\left(\left\langle s_{1}, s_{2}\right\rangle, N, P\right) \leq d c\left(\left\langle s_{1}^{\prime}, s_{2}^{\prime}\right\rangle, N, P\right)$ is satisfied.

For QIS, we only need to prove that for all $c \in \operatorname{cost}\left(\left\langle s_{1}, s_{2}\right\rangle, N, g,\left\langle\hat{s}_{1}, \hat{s}_{2}\right\rangle\right)$, there exists $c^{\prime} \in \operatorname{cost}\left(\left\langle s_{1}^{\prime}, s_{2}^{\prime}\right\rangle, N, g,\left\langle\hat{s}_{1}^{\prime}, \hat{s}_{2}^{\prime}\right\rangle\right)$, such that $\left\langle\hat{s}_{1}, \hat{s}_{2}\right\rangle \lesssim\left\langle\hat{s}_{1}^{\prime}, \hat{s}_{2}^{\prime}\right\rangle$ and $c \leq c^{\prime}$. Since $c \in \operatorname{cost}\left(\left\langle s_{1}, s_{2}\right\rangle, N, g,\left\langle\hat{s}_{1}, \hat{s}_{2}\right\rangle\right)$, according to Definition 2, there exist $c_{1}, c_{2}$ such that $c=c_{1} \oplus c_{2}$ and $s_{1} \stackrel{N_{1}, g_{1}, c_{1}}{\longrightarrow} \mathscr{P}_{1} \quad \hat{s}_{1}$ and $s_{2} \stackrel{N_{2}, g_{2}, c_{2}}{\longrightarrow} \mathscr{P}_{2} \quad \hat{s}_{2}$, where $N_{1} \cup N_{2}=N$, $g_{1} \wedge g_{2}=g$. Because $s_{1} \lesssim s_{1}^{\prime}, s_{2} \lesssim s_{2}^{\prime}$, there always exist transitions $s_{1}^{\prime} \stackrel{N_{1}, g_{1}, c_{1}^{\prime}}{\longrightarrow} \mathscr{Q}_{1} \hat{s}_{1}^{\prime}$ and $s_{2}^{\prime} \stackrel{N_{2}, g_{2}, c_{2}^{\prime}}{\longrightarrow} \mathscr{Q}_{2} \hat{s}_{2}^{\prime}$, where $c_{1} \leq c_{1}^{\prime}$ and $c_{2} \leq c_{2}^{\prime}$. Due to the distributivity of $\oplus$ over $\oplus$ in Q-algebra, we have $c \leq c_{1}^{\prime} \oplus c_{2}^{\prime}$ where $c_{1}^{\prime} \oplus c_{2}^{\prime} \in \operatorname{cost}\left(\left\langle s_{1}^{\prime}, s_{2}^{\prime}\right\rangle, N, g,\left\langle\hat{s}_{1}^{\prime}, \hat{s}_{2}^{\prime}\right\rangle\right)$.

The proof for weak simulation is similar like simulation.

To see that the compositionality does not hold for weak quality improving simulation, consider the QCA given as follows where $R=\left(\mathbb{N}_{+} \cup\{\infty\}, \max ,+,+, \infty, 0\right)$ :

$$
\begin{aligned}
& \text { - } \mathscr{P}_{1}=\left(\left\{s_{0}, s_{1}, s_{2}\right\}, s_{0}, N, R, \longrightarrow\right) \text {, in which } s_{0} \stackrel{\tau, 12}{\longrightarrow} s_{1} \text { and } s_{1} \stackrel{N, g, 5}{\longrightarrow} s_{2}, \\
& \text { - } \mathscr{P}_{2}=\left(\left\{s_{0}^{\prime}, s_{1}^{\prime}, s_{2}^{\prime}\right\}, s_{0}^{\prime}, N, R, \longrightarrow\right) \text {, in which } s_{0}^{\prime} \stackrel{\tau, 2}{\longrightarrow} s_{1}^{\prime} \text { and } s_{1}^{\prime} \stackrel{N, g, 9}{\longrightarrow} s_{2}^{\prime}, \\
& \text { - } \mathscr{Q}_{1}=\left(\left\{t_{0}, t_{1}, t_{2}\right\}, t_{0}, N, R, \longrightarrow\right) \text {, in which } t_{0} \stackrel{\tau, 10}{\longrightarrow} t_{1} \text { and } t_{1} \stackrel{\frac{N, g}{\longrightarrow}}{\longrightarrow} t_{2}, \\
& \text { - } \mathscr{Q}_{2}=\left(\left\{t_{0}^{\prime}, t_{1}^{\prime}, t_{2}^{\prime}\right\}, t_{0}^{\prime}, N, R, \longrightarrow\right) \text {, in which } t_{0}^{\prime} \stackrel{N, g}{\longrightarrow} t_{1}^{\prime} \text { and } t_{1}^{\prime} \stackrel{N, 9}{\longrightarrow} t_{2}^{\prime} .
\end{aligned}
$$

According to Definition 2 and 8 we have both $\mathscr{P}_{1} \lesssim w q \mathscr{Q}_{1}$ and $\mathscr{P}_{2} \lesssim w q \mathscr{Q}_{2}$. However, we have $\left\langle s_{0}, s_{0}^{\prime}\right\rangle \stackrel{N, g, 23}{\longrightarrow}\left\langle s_{2}, s_{2}^{\prime}\right\rangle$ in $\mathscr{P}_{1} \bowtie \mathscr{P}_{2}$ and $\left\langle t_{0}, t_{0}^{\prime}\right\rangle \stackrel{N, g, 21}{\longrightarrow}\left\langle t_{2}, t_{2}^{\prime}\right\rangle$ in $\mathscr{Q}_{1} \bowtie \mathscr{Q}_{2}$ respectively. Therefore, the weak quality improving simulation does not hold between $\left\langle s_{0}, s_{0}^{\prime}\right\rangle$ and $\left\langle t_{0}, t_{0}^{\prime}\right\rangle$.

On the other hand, the hiding operator preserves all kinds of simulation preorders. 
Theorem 2. If $\mathscr{P} \lessdot \mathscr{Q}$, where $\lessdot$ is any kind of simulation relation, then for a given set of nodes $M$, hide $(\mathscr{P}, M) \lessdot \operatorname{hide}(\mathscr{Q}, M)$.

Proof: To prove the result, it suffices to show that for a QCA $\mathscr{Q}=\left(S, s_{0}, \mathscr{N}, R, \longrightarrow\right)$, and $M$, any (weak / quality improving / weak quality improving) simulation relation $\mathscr{R}$ for $\mathscr{Q}$ is a (weak / quality improving / weak quality improving) simulation relation for hide $(\mathscr{Q}, M)$. We first consider the simulation case. Let $\rightsquigarrow$ be the relation such that $s \rightsquigarrow s^{\prime}$ iff there exists a finite path

$$
s \stackrel{M, g_{1}, c_{1}}{\longrightarrow} s_{1} \stackrel{M, g_{2}, c_{2}}{\longrightarrow} s_{2} \ldots \stackrel{M, g_{n}, c_{n}}{\longrightarrow} s^{\prime}
$$

where all the $g_{i}$ s are satisfiable. Then by considering the $M$-transitions in $\mathscr{Q}$, we obtain

$$
\left(s_{1}, s_{2}\right) \in \mathscr{R} \wedge s_{1} \rightsquigarrow s_{1}^{\prime} \Rightarrow s_{2} \rightsquigarrow s_{2}^{\prime} \text { for some state } s_{2}^{\prime}
$$

where $\left(s_{1}^{\prime}, s_{2}^{\prime}\right) \in \mathscr{R}$. Let $\left(s_{1}, s_{2}\right) \in \mathscr{R}, N$ a nonempty subset of $\mathscr{N} \backslash M$, and $P$ an $\mathscr{R}$-upward closed subset of $\mathscr{Q}$. Then for all states $s$ of $\mathscr{Q}$,

$$
d c_{\text {hide }(\mathscr{Q}, M)}(s, N, P)=\bigvee_{s^{\prime} \in s^{*}}\left(d c_{\mathscr{Q}}\left(s^{\prime}, N, P\right) \vee d c_{\mathscr{Q}}\left(s^{\prime}, N \cup M, P\right)\right)
$$

where $s^{*}=\left\{s^{\prime} \in S: s \rightsquigarrow s^{\prime}\right\}$. From (1) we obtain that for every $s_{1}^{\prime} \in s_{1}^{*}$ there exists a state $s_{2}^{\prime} \in s_{2}^{*}$ with $\left(s_{1}, s_{2}\right) \in \mathscr{R}$. Because

$$
\begin{aligned}
d c_{\mathscr{Q}}\left(s_{1}^{\prime}, N, P\right) & \leq d c_{\mathscr{Q}}\left(s_{2}^{\prime}, N, P\right) \\
d c_{\mathscr{Q}}\left(s_{1}^{\prime}, N \cup M, P\right) & \leq d c_{\mathscr{Q}}\left(s_{2}^{\prime}, N \cup M, P\right)
\end{aligned}
$$

we get $d c_{\text {hide }(\mathscr{Q}, M)}\left(s_{1}, N, P\right) \leq d c_{\text {hide }(\mathscr{Q}, M)}\left(s_{2}, N, P\right)$.

The proof for weak simulation is similar like simulation.

Now we consider the case for quality improving simulation. If $\left(s_{1}, s_{2}\right) \in \mathscr{R}$, then

$$
\forall c \in \operatorname{cost}\left(s_{1}, N, g, s_{1}^{\prime}\right) . \exists c^{\prime} \in \operatorname{cost}\left(s_{2}, N, g, s_{2}^{\prime}\right) . s . t .\left(s_{1}^{\prime}, s_{2}^{\prime}\right) \in \mathscr{R} \wedge c \leq c^{\prime}
$$

Whenever $N \backslash M \neq \emptyset$, we can easily get

$\forall c \in \operatorname{cost}_{\mathrm{hide}(\mathscr{Q}, M)}\left(s_{1}, N \backslash M, \exists M[g], s_{1}^{\prime}\right) . \exists c^{\prime} \in \operatorname{cost}_{\mathrm{hide}(\mathscr{Q}, M)}\left(s_{2}, N \backslash M, \exists M[g], s_{2}^{\prime}\right)$. s.t. $\left(s_{1}^{\prime}, s_{2}^{\prime}\right) \in \mathscr{R} \wedge c \leq c^{\prime}$

Therefore, by considering the proof for simulation, we know that for any transition $s_{1} \stackrel{\hat{N}, \hat{g}, c}{\longrightarrow} s_{1}^{\prime}$ in $\operatorname{hide}(\mathscr{Q}, M)$ with cost $c$ and $\left(s_{1}, s_{2}\right) \in \mathscr{R}$, there always exist a transition $s_{2} \stackrel{\hat{N}, \hat{g}, c^{\prime}}{\longrightarrow} s_{2}^{\prime}$ such that the condition for quality improving simulation is still staisfied in $\operatorname{hide}(\mathscr{Q}, M)$. So $\mathscr{R}$ is still a quality improving simulation in hide $(\mathscr{Q}, M)$.

For weak quality improving simulation, suppose $\left(s_{1}, s_{2}\right) \in \mathscr{R}$, then we have

$$
\forall c \in \operatorname{cost}_{w}\left(s_{1}, N, g, s_{1}^{\prime}\right) . \exists c^{\prime} \in \operatorname{cost}_{w}\left(s_{2}, N, g, s_{2}^{\prime}\right) . \text { s.t. }\left(s_{1}^{\prime}, s_{2}^{\prime}\right) \in \mathscr{R} \wedge c \leq c^{\prime}
$$

If $N \backslash M=\emptyset$, suppose $s_{1}^{\prime} \stackrel{\hat{N}, \hat{g}, \hat{c}}{\longrightarrow} s_{1}^{\prime \prime}$, and $\hat{N} \backslash M \neq \emptyset$, then we have $s_{2}^{\prime} \stackrel{\hat{N}, \hat{g}, \hat{c}^{\prime}}{\longrightarrow} s_{2}^{\prime \prime}$ where $\left(s_{1}^{\prime \prime}, s_{2}^{\prime \prime}\right) \in \mathscr{R}$ and $\hat{c} \leq \hat{c}^{\prime}$. Therefore, in hide $(\mathscr{Q}, M)$, there exist transitions $s_{1} \stackrel{\tau, c}{\longrightarrow}$ 
$s_{1}^{\prime}$ and $s_{2} \stackrel{\tau, c^{\prime}}{\longrightarrow} s_{2}^{\prime}$, and thus weak atomic runs $s_{1} \stackrel{\hat{N}, \hat{g}, c \otimes \hat{c}}{\longrightarrow} s_{1}^{\prime \prime}$ and $s_{2} \stackrel{\hat{N}, \hat{g}, c^{\prime} \otimes \hat{c}^{\prime}}{\longrightarrow} s_{2}^{\prime \prime}$. According to the definition of Q-algebra, $\otimes$ distributes over $\oplus$, i.e.,

$$
a \otimes(c \oplus d)=(a \otimes c) \oplus(a \otimes d)
$$

So if $c \leq c^{\prime}, \hat{c} \leq \hat{c}^{\prime}$, then $c \otimes \hat{c} \leq c^{\prime} \otimes \hat{c}^{\prime}$. Therefore, the condition for weak quality improving simulation is still staisfied in $\operatorname{hide}(\mathscr{Q}, M)$.

\section{Conclusion}

In this paper we introduced QCA as an operational model for reasoning about component connectors with QoS guarantees, together with notions of simulation that are preserved by the QCA product. This result together with the relationship between weak and strong version of (quality improving) simulations provide the basis for analysis of both functional and non-functional aspects of Reo component connectors.

In our future activity, we will work on the development of appropriate logic and quantitative model checking algorithms based on the QCA model, and investigate the expressiveness of our model and its relationship with other extensions to constraint automata, such as in $[3,5,7]$. Reo and constraint automata have been successfully applied in web service composition [17], but whether the QCA model can be taken as a new, proper entry in the bazaar of web service composition with QoS constraints [16], is still an open research question.

Acknowledgements. The authors are indebted to R. D. van der Mei, C. G. Verhoef, and the members of SEN3 for helpful discussions. We are also grateful to the referees for their constructive criticism.

\section{References}

1. F. Arbab. Abstract Behavior Types: A Foundation Model for Components and Their Composition. In F. S. de Boer, M. M. Bonsangue, S. Graf, and W.-P. de Roever, editors, Formal Methods for Components and Objects: First International Symposium, FMCO 2002, Leiden, The Netherlands, November 2002, Revised Lectures, volume 2852 of LNCS, pages 33-70. Springer, 2003.

2. F. Arbab. Reo: A Channel-based Coordination Model for Component Composition. Mathematical Structures in Computer Science, 14(3):329-366, 2004.

3. F. Arbab, C. Baier, F. de Boer, and J. Rutten. Models and Temporal Logics for Timed Component Connectors. In J. R. Cuellar and Z. Liu, editors, SEFM2004, 2nd International Conference on Software Engineering and Formal Methods, pages 198-207. IEEE Computer Society, 2004.

4. F. Arbab and J. Rutten. A coinductive calculus of component connectors. In M. Wirsing, D. Pattinson, and R. Hennicker, editors, Recent Trends in Algebraic Development Techniques: 16th International Workshop, WADT 2002, Frauenchiemsee, Germany, September 24-27, 2002, Revised Selected Papers, volume 2755 of LNCS, pages 34-55. Springer-Verlag, 2003. 
5. C. Baier. Probabilistic Models for Reo Connector Circuits. Journal of Universal Computer Science, 11(10):1718-1748, 2005.

6. C. Baier, M. Sirjani, F. Arbab, and J. Rutten. Modeling component connectors in Reo by constraint automata. Science of Computer Programming, 61:75-113, 2006.

7. C. Baier and V. Wolf. Stochastic Reasoning About Channel-Based Component Connectors. In P. Ciancarini and H. Wiklicky, editor, COORDINATION 2006, volume 4038 of LNCS, pages 1-15. Springer-Verlag, 2006.

8. M. Bernardo and R. Gorrieri. A tutorial on EMPA: a theory of concurrent processes with nondeterminism, priorities, probabilities and time. Theoretical Computer Science, 202:1-54, 1998.

9. S. Bistarelli, U. Montanari, and F. Rossi. Semiring-based constraint satisfaction and optimization. JACM, 44(2):201-236, 1997.

10. T. Chothia and J. Kleijn. Q-Automata: Modelling the Resource Usage of Concurrent Components. In Proceedings of FOCLASA 2006, ENTCS, 2006.

11. N. Götz, U. Herzog, and M. Rettelbach. Multiprocessor and distributed system design: the integration of functional specification and performance analysis using stochastic process algebras. In L. Donatiello and R. Nelson, editors, Performance Evaluation of Computer and Communication Systems, volume 729 of LNCS, pages 121-146. Springer, 1993.

12. H. Hermanns. Interactive Markov Chains And the Quest for Quantified Quality, volume 2428 of $L N C S$. Springer, 2002.

13. J. Hillston. A Compositional Approach to Performance Modelling. Cambridge University Press, 1996.

14. M. P. Papazoglou and D. Georgakopoulos. Service Oriented Computing. Comm. ACM, 46(10):25-28, 2003.

15. M. A. Marsan, G. Conte, and G. Balbo. A Class of Generalized Stochastic Petri Nets for the Performance Evaluation of Multiprocessor Systems. ACM Transactions on Computer Systems, 2(2):93-122, 1984.

16. D. A. Menascé. Composing Web Services: A QoS View. IEEE Internet Computing, 8(6):8890, 2004.

17. S. Meng and F. Arbab. Web Services Choreography and Orchestration in Reo and Constraint Automata. In Proceedings of SAC'07, 2007.

18. R. Milner. An algebraic definition of simulation between programs. In D. C. Cooper, editor, Proceedings of the 2nd International Joint Conference on Artifial Intelligence, London, UK. William Kaufmann, British Computer Society, 1971.

19. M. Mohri, F. Pereira, and M. Riley. Weighted automata in text and speech processing. In ECAI'96 Workshop on Extended Finite State Models of Language, pages 46-50, 1996.

20. R. D. Nicola, G. Ferrari, U. Montanari, R. Pugliese, and E. Tuosto. A process calculus for qos-aware applications. In Coordination 2005, volume 3454 of LNCS, pages 33-48. Springer, 2005. 